\title{
Analysis of Incomplete Filling Defect for Injection-Molded Air Cleaner Cover Using Moldflow Simulation
}

\author{
Hyeyoung Shin ${ }^{1}$ and Eun-Soo Park ${ }^{2}$ \\ ${ }^{1}$ Kumho Petrochemical R\&D Center, Hwaam-Dong 57-1, Yuseong, Daejeon 305-348, Republic of Korea \\ ${ }^{2}$ Youngchang Silicone Co., Ltd., 481-7 Gasan-Dong, Kumchun-Gu, Seoul 153-803, Republic of Korea \\ Correspondence should be addressed to Eun-Soo Park; t2phage1@daum.net
}

Received 22 March 2013; Revised 21 May 2013; Accepted 10 June 2013

Academic Editor: Pasquale Longo

Copyright (c) 2013 H. Shin and E.-S. Park. This is an open access article distributed under the Creative Commons Attribution License, which permits unrestricted use, distribution, and reproduction in any medium, provided the original work is properly cited.

\begin{abstract}
A large-sized cover part for air cleaner was injection molded with ABS resin, and its incomplete filling defect was analyzed using commercial Moldflow software. To investigate the effect of processing temperature on incomplete filling defect, tensile properties, weight loss, and phase separation behavior of ABS resin were evaluated. The tensile properties of dumbbell samples were not changed up to $250^{\circ} \mathrm{C}$ and decreased significantly thereafter. SEM micrographs indicated no significant changes in the status of polybutadiene rubber phase below $250^{\circ} \mathrm{C}$. These different test results indicated that ABS resin little affected the thermal decomposition in processing temperature range. The Moldflow simulation was performed using measured thickness of molded cover and actual mold design with the defects. As expected, the cover part showed unbalanced filling and incomplete sections. To improve these defects, two possible cases of hot runner system have been simulated. When applying modified 5-gate system, the maximum injection pressure was decreased approximately 5.5\% more than that of actual gate system. In case of 6-gate system, the maximum injection pressure reduced by $23 \%$, and the injection pressure required to fill is well within the range of the molding equipment. The maximum clamping force of 6 -gate system was also significantly reduced than that of actual and 5-gate system.
\end{abstract}

\section{Introduction}

Injection molding is one of the most commonly used manufacturing processes for the fabrication of plastic parts in net shape with excellent dimensional tolerance [1]. A wide variety of products are manufactured using injection molding, which vary greatly in their size and complexity [2-5]. Examples of common, everyday products that require plastic injection molding include automobile bumpers, mobile phone housings, television cabinets, compact discs, toys, and lunch boxes are all examples of injection-molded parts. This process requires the use of an injection-molding machine, raw plastic material, and a mold. A plastic material is melted in the injection-molding machine and then injected into the mold, where it cools and solidifies into the final part. The most commonly used thermoplastics are polystyrene, polypropylene, polyvinyl chloride, and acrylonitrile-butadiene-styrene (ABS) [6-9]. ABS resins are among the most versatile thermoplastics in the styrenic polymers. The primary features and benefits of ABS are derived from the tree building blocks. Thermal stability and chemical resistance are derived from acrylonitrile, while butadiene provides impact resistance and toughness. Styrene imparts rigidity and processability to ABS. Therefore, they have practical toughness, high modulus, dimension stability, and good aesthetics for broad range of applications [9-11].

Injection-molding process is a complex technology with possible production problems. They can either be caused by defects in the molds or more often by part processing. Many processing factors are involved in this process and have a great influence on the quality of final products. These factors can be usually classified into four categories: materials, injection machine, model design, and process conditions [10]. Although the process and stages of custom injection molding can be as quick as just a few seconds, precision and accuracy are critical when it comes to such condition factors as time, pressure, temperature, and force. Possible defects that can occur in a molded plastic product include flash, which occurs 
when the clamp force is too low and molten material seeps out of the molding area and warping and unfilled sections. Each factor, accurately chosen and controlled, requires a multidisciplinary knowledge in order to improve and optimize final product performances.Simulation of injection molding is widely used to help mold designers to select appropriate injection technology and investigate product performance and processability [2-6]. It is the most successful example of simulation for any plastic forming process. Benefits that have been identified from experienced users of analysis programmed are better part quality, reduced tooling lead times, optimum cycle times, and reduce rework and scrapping of materials [3]. This may be contrasted with other polymer forming processes where tooling costs are much lower or where problems may be overcome by varying process conditions. Therefore, the simulation is usually conducted during the product design stage and the early stages of the tool design.

Many simulation studies were performed to determined potential problems and their most economical solutions. Shen et al. [2] have done numerical analysis on a thin walled part which has a fiber reinforced thermoplastic material. Moldflow software was used for the analysis. Different injection parameters (injection pressure, melt temperature, mold temperature, injection time), different fibers, different fiber ratios, and different thickness values were used. The outer shell of a notebook computer was used as an analysis model. Dairanieh et al. [4] examine the sensitivity of Moldflow's weld-line prediction algorithm to variations in material properties and processing conditions. Qualitatively, the algorithm correctly predicted the effects of changes in viscosity, density, and pressure-viscosity-temperature (PVT) relationship on weld-line strength of a poly(methyl methacrylate). Huang and Tai [5] have examined the influential effects over warpage that is seen in thin walled parts produced by injection molding. C-Mold software was used for the analysis. They have used the Taguchi experimental method to decide the injection parameters. As a result, packing pressure was found as the most important factor that affects warpage. It is seen that gate location and filling time have little effect over warpage. Shin and Park [6] have examined the residual stresses in a part which was produced by plastic injection method using Moldflow software. For analysis, screw of ice water filter machine was used. They found that the majority of the crack comes from residual stress in the final part that originated from higher injection pressure during injectionmolding process.

In this study, a large-sized cover for air cleaner was injection molded with ABS resin and its incomplete filling defect was analyzed. During the manufacturing of cover, many undesired products with unfilled sections and burrs were formed repeatedly. The incomplete filling is one type of the defect that normally will appear either due to a lack of fluidity in the molten polymer or cross-section of mold that is too narrow. To determine the effect of processing temperature on these defects, the weight loss, phase separation behavior, and tensile properties of ABS resin which was used in manufacturing were investigated. At the same time, Moldflow simulation was conducted using actual mold used in
TABLE 1: ABS specifications.

\begin{tabular}{lcc}
\hline Properties & Unit & Values \\
\hline Density & $\mathrm{g} / \mathrm{cm}^{3}$ & 1.04 \\
Rockwell hardness & $R$ scale & 108 \\
& $\mathrm{~g} / 10 \mathrm{~min}$ & $7\left(200^{\circ} \mathrm{C}, 5.0 \mathrm{~kg}\right)$ \\
Melt index & $\mathrm{g} / 10 \mathrm{~min}$ & $50\left(220^{\circ} \mathrm{C}, 10.0 \mathrm{~kg}\right)$ \\
& $\mathrm{g} / 10 \mathrm{~min}$ & $75\left(200^{\circ} \mathrm{C}, 21.6 \mathrm{~kg}\right)$ \\
Heat distortion temperature & ${ }^{\circ} \mathrm{C}$ & $85\left(18.6 \mathrm{~kg} / \mathrm{cm}^{2}\right)$ \\
Vicat softening point & ${ }^{\circ} \mathrm{C}$ & 85 \\
\hline
\end{tabular}

manufacturing process. For the injection-molding simulation analysis, polymer rheological properties are one of the vital data that must be taken into consideration. Accordingly, the viscosity and PVT measurement of ABS resin were also performed on a capillary rheometer and on a high-pressure mercury dilatometer, respectively. Analyzing the defect leads to identifying the root cause of the defect and to finding the solution to overcome the defect in further development processes. Finally, two case studies are demonstrated to show the feasibility of the proposed optimization methods.

\section{Experimental}

2.1. Materials. ABS resin (ABS 780, Kumho Petrochemical Co., Seoul, Korea) was used as received. ABS was predried in a convection oven for at least $12 \mathrm{~h}$ at $80^{\circ} \mathrm{C}$ to remove any moisture from the pellets before processing. The characteristics of the resin used in this study are summarized in Table 1.

2.2. Instrumentation. Scanning electron microscopy (SEM) observations of the samples were performed on a Hitachi S-4300 model (Tokyo, Japan). The fractured surfaces of the composites were prepared using cryogenic fracturing in liquid nitrogen followed by a coating with platinum in an SPI sputter coater. The morphology was determined using an accelerating voltage of $15 \mathrm{kV}$.

The surface appearance of samples was measured with a video microscope (Inf-500TM, charge-coupled device (CCD) video microscope, Moritex Corp., Tokyo, Japan) with a PC that contained video capture software (i-Solution Lite, Image \& Microscope Technology, Daejeon, Korea).

2.3. Tensile Test Procedure. ABS sheets (ABS 780 pellets) were prepared on a hot press at $220,230,240,250$, and $260^{\circ} \mathrm{C}$ for 20 min under ca. 5 atm, followed by quenching in water. The sheet (around $1.5 \mathrm{~mm}$ ) thus formed was free from any distortion problems. Dumbbell specimens for mechanical tests were prepared in accordance with IEC 60811-1-1 specification. The test specimens were preconditioned to $20 \%$ relative humidity and $20 \pm 1^{\circ} \mathrm{C}$ in order to standardize test conditions. Tensile properties of samples were determined with a universal test machine (UTM, Model DECMC00, Dawha test machine, Korea) at a cross-head speed of $50 \mathrm{~mm} / \mathrm{min}$. The mean value of at least five specimens of each sample was 
taken, although specimens that broke in an unusual manner were disregarded.

2.4. Weight Loss Test. ABS pellets were thermal aged in dry oven maintained at $230-260^{\circ} \mathrm{C}$. At predetermined intervals, specimens were removed from the dry oven and cooled to room temperature until constant weight. The cooled specimens were weighed to calculate the weight loss. Five measurements were taken on average.

Sample surface after the weight loss test were measured by a video microscope (Inf-500TM CCD Video Microscope, Moritex Corp., Japan) with a PC that contained video capture software (i-Solution Lite, Image and Microscope Technology, Korea).

2.5. Injection Molding of Cover. Cover part for air cleaner was injection molded at a speed of $100 \mathrm{rpm}$ using a 2,500ton injection-molding machine (ND2500 (screw diameter: $\phi 140$, maximum injection pressure: $1,640 \mathrm{~kg} / \mathrm{cm}^{2}$, maximum injection rate: $1,370 \mathrm{~cm}^{3} / \mathrm{s}$ ), Woojin Selex Co., Ltd., Anyang, Korea) equipped with a 1:5 compression ratio screw using a temperature setting of rear zone: $205^{\circ} \mathrm{C}$, second zone: $225^{\circ} \mathrm{C}$, and forward zone: $230-260^{\circ} \mathrm{C}$. The processing parameters used in manufacturing are summarized in Table 2.

2.6. Shear Viscosity. Viscosity measurement of pristine ABS was performed on capillary rheometer (RH10, Malvern Instruments Ltd., Worcestershire, UK) with the length-toinner diameter (L/D) of the circular die was 20/1. Studies were done at four test temperatures of $200,220,240$, and $260^{\circ} \mathrm{C}$, and the range of shear rates varied from 1 to $8000 \mathrm{~s}^{-1}$.

2.7. Pressure-Volume-Temperature (PVT) Measurement. The PVT behavior was investigated on a high-pressure mercury dilatometer GNOMIX (Gnomix, Inc., USA) in pressure range $50-200 \mathrm{MPa}$ at temperatures $25-260^{\circ} \mathrm{C}$ under isobaric conditions. The heating and cooling rates of $1^{\circ} \mathrm{C} / \mathrm{min}$ were selected for experimental runs.

2.8. Moldflow Analysis. Moldflow (Moldflow 6.2, Moldflow Corporation, MA, USA) simulation was conducted using a single cavity mold with hot runner gating system. The 3D-CAD drawing of cover mold used in manufacturing was exported to the FE software using STEP file format. Therefore, mold cavity has exactly the same geometries as the manufacturing. Several iterations were carried out to establish optimum location of the sprue gate in order to achieve the most balanced flow into the cavity along with uniform pressure drop and flow front temperature. All simulations were separately carried out for each component by using configurations with five or six gates, and the process parameters are reported in Table 2. The hot runner diameter was $12 \mathrm{~mm}$, and the circular sprue diameter was tapered from 12 to $6 \mathrm{~mm}$. The sprue length and gate diameter were 50 and $10 \mathrm{~mm}$, respectively.
TABLE 2: Processing parameters used in the manufacturing and Moldflow simulation.

\begin{tabular}{lccc}
\hline $\begin{array}{l}\text { Processing } \\
\text { parameters }\end{array}$ & Unit & $\begin{array}{c}\text { Manufacturing } \\
\text { values }\end{array}$ & Simulation values \\
\hline Flow rate & $\mathrm{cm}^{3} / \mathrm{s}$ & $1,000-1200$ & 1,000 \\
Melt temperature & ${ }^{\circ} \mathrm{C}$ & $230-260$ & 230 \\
Mold temperature & ${ }^{\circ} \mathrm{C}$ & \multicolumn{2}{c}{40} \\
Filling/packing & - & Switch over at 98 vol $\%$ \\
Packing profile & - & $80 \%$ of maximum pressure \\
Holding time & $\mathrm{s}$ & \multicolumn{2}{c}{10} \\
Cooling times & $\mathrm{s}$ & \multicolumn{2}{c}{20} \\
\hline
\end{tabular}

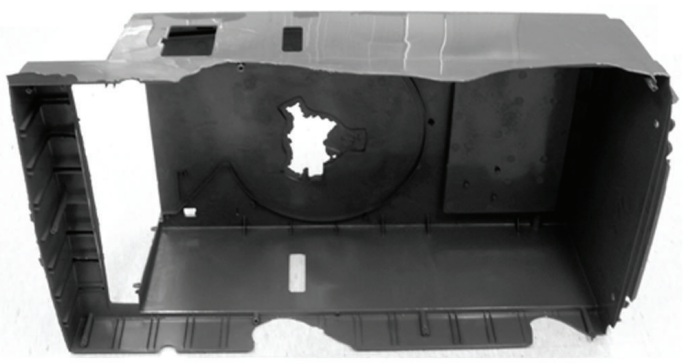

FIGURE 1: Image of the inferior cover part.

\section{Results and Discussion}

3.1. Problem Statements. The cover part for air cleaner was injection molded with $\mathrm{ABS}$ resin using a 2,500-ton injectionmolding machine. The main dimension of cover was 1,070 $\times 580 \times 520 \mathrm{~mm}^{3}$ and the average thickness of component was $3.0 \mathrm{~mm}$. During manufacturing process, many undesired products were formed repeatedly, and a representative image of inferior product was demonstrated in Figure 1. It can be seen that the cover has many unfilled sections and burrs.

Injection molding is a cyclic process. A typical injectionmolding process consists of four stages: (i) filling of the molten polymer into the mold; (ii) packing of more material into the mold under high pressure to compensate for volumetric shrinkage of the material as it cools; (iii) cooling during which the material solidifies while in the mold until it is sufficiently solid; (iv) ejection of the solidified product from the mold [12].

The incomplete filling defects are caused by a wrong injection-molding temperature, resin burnout, insufficient shot volume, or low flow rate of molten plastics. During the filling stage, the hot molten polymer must fill the cold mold completely before it solidifies. It can be assumed that the processing temperature and the flow rate of molten polymer are maintained too high to eliminate unfilled sections and these lead to additional burr defect.

3.2. Injection-Molding Conditions. Computer analysis of the filling and packing phases of injection-molding process needs data on the physical properties, mechanical properties, and 
rheological properties of polymer is constructed and the material from which the mold is also constructed [13].

Table 3 summarized the effect of processing temperature on the tensile properties of ABS resin. The abbreviation of the sample code in Table $3, \mathrm{ABS}-220^{\circ} \mathrm{C}$, for example, means that the ABS was press-molded at $220^{\circ} \mathrm{C}$ for $20 \mathrm{~min}$. The tensile properties of dumbbell samples were not changed up to $250^{\circ} \mathrm{C}$ and decrease significantly thereafter. In case of ABS $-260^{\circ} \mathrm{C}$, an approximately 56 and $40 \%$ decrease in the tensile strength and elongation at break of $190 \mathrm{~kg}_{\mathrm{f}} / \mathrm{cm}^{2}$ and $3 \%$ were observed compared to $\mathrm{ABS}-220^{\circ} \mathrm{C}$, respectively.

The significant drop in tensile properties at higher processing temperature can be related to the increase in brittleness of ABS specimens driven by chemical changes in the polybutadiene (PB) microstructure, which are attributed to chain scission and cross-linking [13]. Thermal degradation follows the free radicals mechanism, initiated by oxygen or oxidative contamination in the material or other stresses. In the initial stages of thermal degradation of polymer, chain scission often prevails, which reduces molecular weight. Furthermore, degradation of the PB phase at the surface forms hydroperoxide species [14-16]. Degradation of PB phase by cross-linking would also reduce polymer chain mobility and decrease free volume [14].

Figure 2 shows the influence of thermal aging at various temperatures on the weight loss of ABS 780 pellets used in manufacturing for cover. The weight loss gradually increased up to $40 \mathrm{~min}$ with the aging time and almost maintained thereafter. It is noted that the weight loss of ABS was drastically increased during thermal aging at $260^{\circ} \mathrm{C}$. Approximately, a minimum of $20 \%$ overall increase in the weight loss was observed comparing to $230^{\circ} \mathrm{C}$. The weight decrease in pellet after thermal aging is attributed to the decrease in low molecular weight components, such as volatile residues, additives, oligomers, and thermal degradation products. There was a good correspondence between the changes in tensile properties and the observed weight loss variations. This is consistent with the changes outlined above, namely, the degradation of the PB phase. Finally, they usually create a weakened pathway within the material by creating permanent molecular or physical changes. This leads to formation of voids (gas porosity) or defects of injection-molding products and lowering the quality of final products.

Figure 3 shows the phase separation behavior of the ABS sheet after the thermal aging at $230,240,250$, and $260^{\circ} \mathrm{C}$ for $20 \mathrm{~min}$. All the samples after thermal aging appeared to have a noticeable increase in white pattern. The white pattern was likely a product of chemical oxidation-induced phase separation by surface directed thermal decomposition and release of internal stress that had accumulated during oxidation. ABS is composed of a two-phase system having a styreneacrylonitrile copolymer (SAN) as the continuous phase with a dispersed phase of $\mathrm{PB}$ rubber. The $\mathrm{PB}$ phase is compatibilized with the rigid SAN component through grafting. Phase separation is induced when a sample is transferred from onephase region into miscibility gap. Usually this is accomplished by a change in temperature, upward or downward depending on the system under study [17]. However, the shape of white

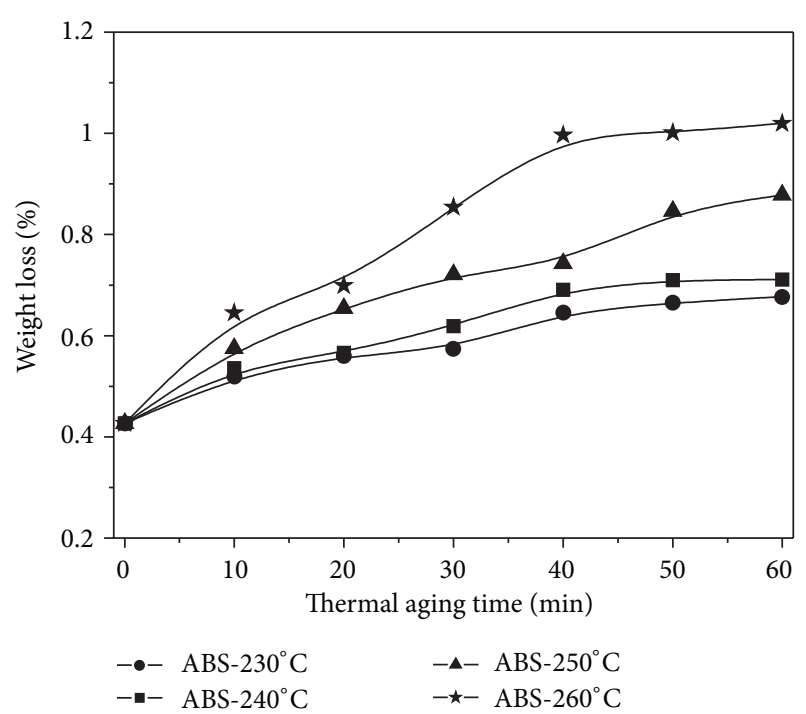

FIGURE 2: Weight loss test results of the ABS resin $\left(t_{0}\right.$ : after drying at $12 \mathrm{~h}$ at $\left.80^{\circ} \mathrm{C}\right)$.

TABLE 3: Tensile properties of the ABS resin.

\begin{tabular}{lcc}
\hline Sample code & \multicolumn{2}{c}{ Tensile properties } \\
& $\begin{array}{c}\text { Tensile strength } \\
\left(\mathrm{kg}_{\mathrm{f}} / \mathrm{cm}^{2}\right)\end{array}$ & $\begin{array}{c}\text { Elongation at break } \\
(\%)\end{array}$ \\
\hline $\mathrm{ABS}-220^{\circ} \mathrm{C}$ & $442 \pm 18$ & $5.0 \pm 0.8$ \\
$\mathrm{ABS}-230^{\circ} \mathrm{C}$ & $445 \pm 31$ & $4.6 \pm 0.7$ \\
$\mathrm{ABS}-240^{\circ} \mathrm{C}$ & $446 \pm 14$ & $4.3 \pm 1.1$ \\
$\mathrm{ABS}-250^{\circ} \mathrm{C}$ & $446 \pm 16$ & $4.3 \pm 0.5$ \\
$\mathrm{ABS}-260^{\circ} \mathrm{C}$ & $190 \pm 16$ & $3.1 \pm 0.7$ \\
\hline
\end{tabular}

patterns was not changed significantly up to $250^{\circ} \mathrm{C}$. From $260^{\circ} \mathrm{C}$ upward, they were gathering together and became more irregular in shape indicated that the phase separation of ABS was accelerated at $260^{\circ} \mathrm{C}$.

It can be also found that yellowing of the ABS specimen started at $250^{\circ} \mathrm{C}$ because it was attacked by heating during thermal aging. Kim and kang have investigated the NMR sequence before and after recycling of ABS specimens. The color of SAN may be varied by the sequence of styrene and acrylonitrile (AN). Yellowing occurs according to the content of sequence AN-AN-AN. However, their study shows that the content of the sequence AN-AN-AN was not much changed by recycling, and the total molecular weight of ABS was not changed [18]. Therefore, the PB component affects the yellowing process which may introduce alterations in the chemical structure of the polymeric chains, resulting in more brittle material with lower ductility [19].

As the thermal aging time increased, phase separation of the $\mathrm{PB}$ phase seems to become progressively more significant (Figure 4). After thermal aging at $260^{\circ} \mathrm{C}$ for $10 \mathrm{~min}$, surface of ABS sample appeared to have a noticeable increase in scratches (Figure 4(b)). With the aging time, the scratches were gathering together and forming wrinkle patterns (Figure $4(\mathrm{c})$ ). Finally, they are arranged in an 


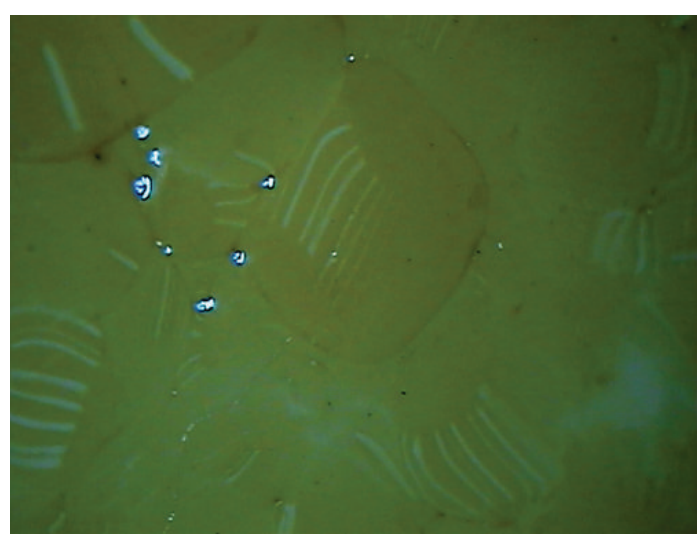

(a)

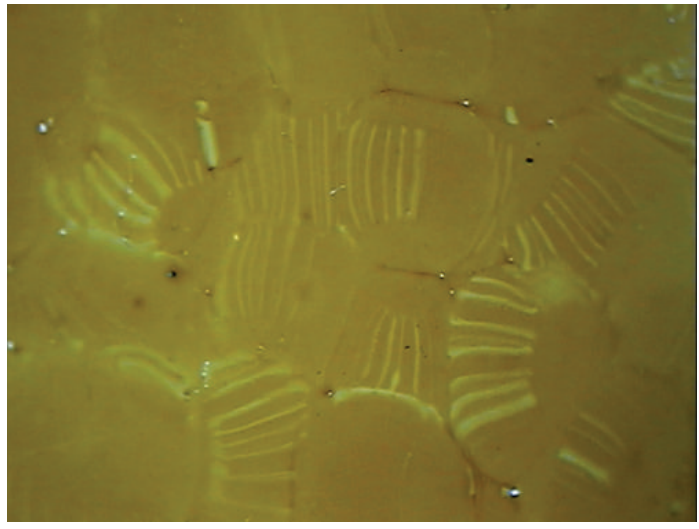

(c)

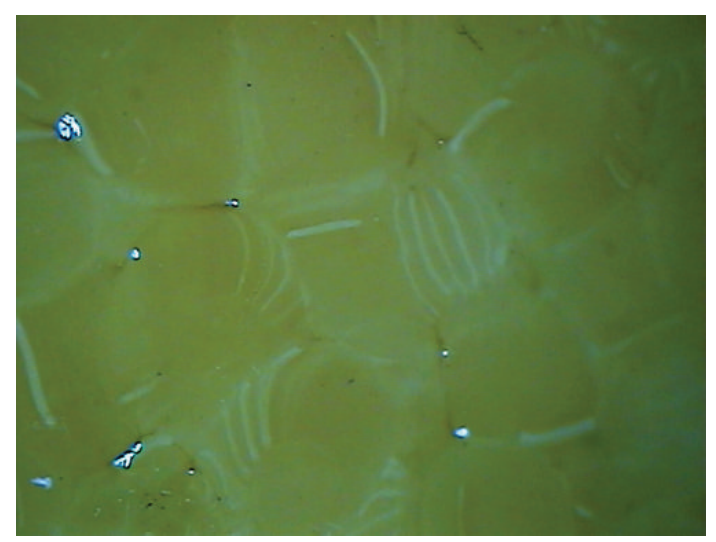

(b)

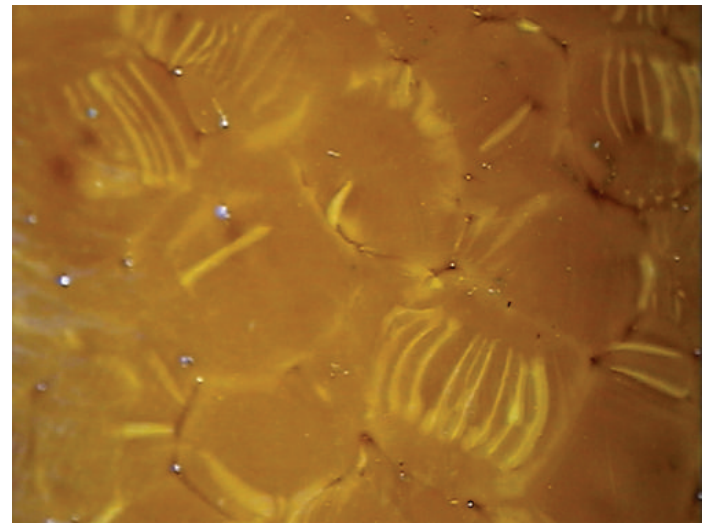

(d)

FIGURE 3: Phase separation phenomenon of the ABS resin after the thermal aging at (a) 230, (b) 240, (c) 250 , and (d) $260^{\circ} \mathrm{C}$ for $1 \mathrm{~h}(20 \mathrm{x})$.

orderly repeating pattern extending in all $\mathrm{PB}$ rubber regions (Figure 4(d)).

Microphase separation in block copolymers is a possible explanation for the pattern formation. In the case of diblock copolymers, consisting of two subchains (a) and (b) made of different monomers $\mathrm{A}$ and $\mathrm{B}$, respectively, even a weak repulsion between unlike monomers induces a strong repulsion between sub-chains. As a result, different sub-chains tend to segregate below some temperature, but as they are chemically bonded, even a complete segregation cannot lead to a macroscopic phase separation as in mixtures of two polymers. Only a local microphase separation occurs, and microphases rich in A or B are formed [15].

The images in Figure 5 demonstrate the fractured surface of the ABS specimen after hot pressing at 220 and $250^{\circ} \mathrm{C}$ for $20 \mathrm{~min}$. In the SEM micrographs, the light regions represent the $\mathrm{PB}$ phase in the ABS, while the dark region represents SAN matrix. The micrographs show that no significant changes in size, shape, and the distribution of the rubber particles. These different test results indicated that ABS resin little affected the thermal decomposition in processing temperature range.

3.3. Viscosity Model. The viscosity is also another important function that needs to provide into the injection moulding flow analysis. The viscosity behavior of ABS resin is obtained by using the Cross-WLF model. Cross-WLF is a well-known rheological model used to predict the viscosities of polymer melts at different temperatures, pressures, and shear rates. This model accounts for the effect of temperature, shear rate, and pressure on the viscosity, over a wide temperature range. The Cross-WLF model is appropriate for postfilling stage, because the temperature and pressure sensitivities of the zeroshear-rate viscosity are well represented [20]. The model is given as

$$
\begin{gathered}
\eta(T, \dot{\gamma})=\frac{\eta_{0}(T)}{1+\left(\eta_{0}(T) \dot{\gamma} / \tau\right)^{1-n}}, \\
\eta_{0}(T)=D 1 \exp \left[\frac{-A 1\left(T-T_{r}\right)}{A 2+\left(T-T_{r}\right)}\right], \\
T_{r}=D 2+D 3 P,
\end{gathered}
$$

where $\eta, \gamma, \tau, T$, and $P$ are the viscosity, shear rate, relaxation time, and pressure, respectively. $T_{r}$ is an arbitrary reference temperature and usually chosen as the material $T_{g} . n, D 1, D 2$, D3, $A 1$, and $A 2$ are model constants.

The ABS resin used in this study has a melt index of $7\left(200^{\circ} \mathrm{C}, 5.0 \mathrm{~kg}\right), 50\left(220^{\circ} \mathrm{C}, 10.0 \mathrm{~kg}\right)$, and $75 \mathrm{~g} / 10 \mathrm{~min}$ 


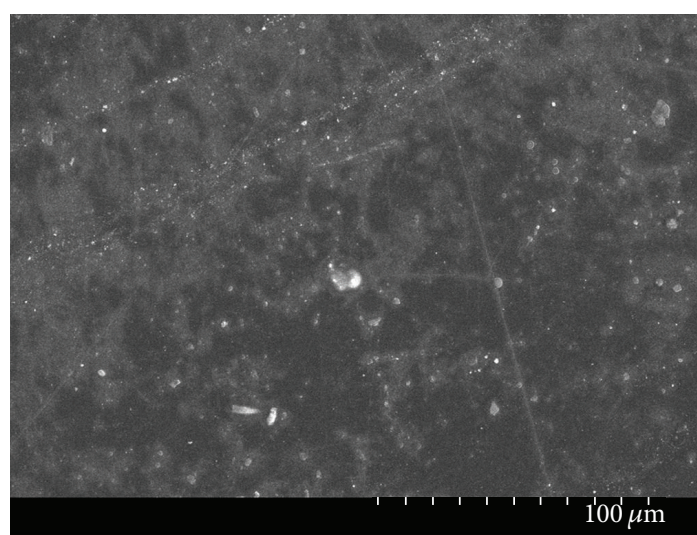

(a)

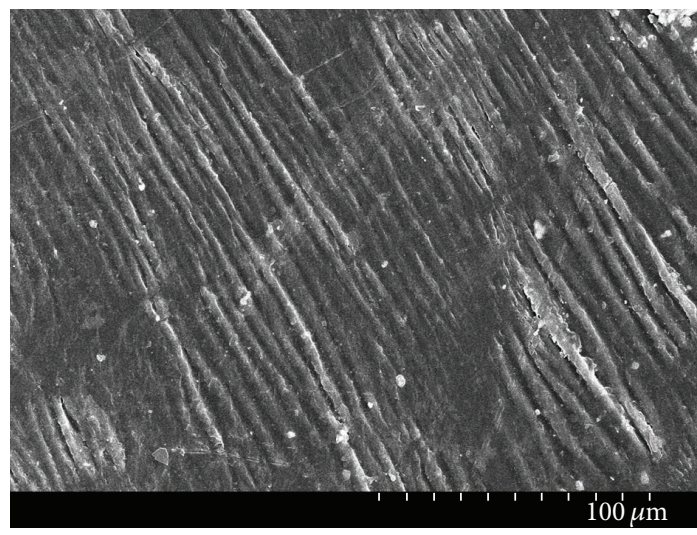

(c)

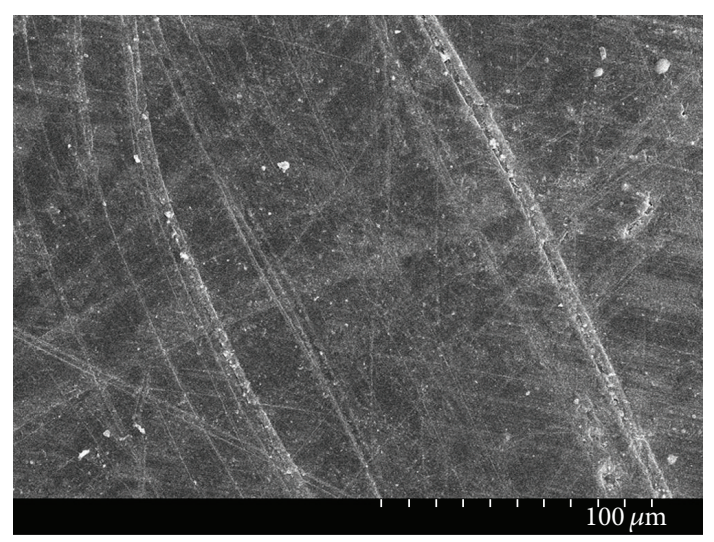

(b)

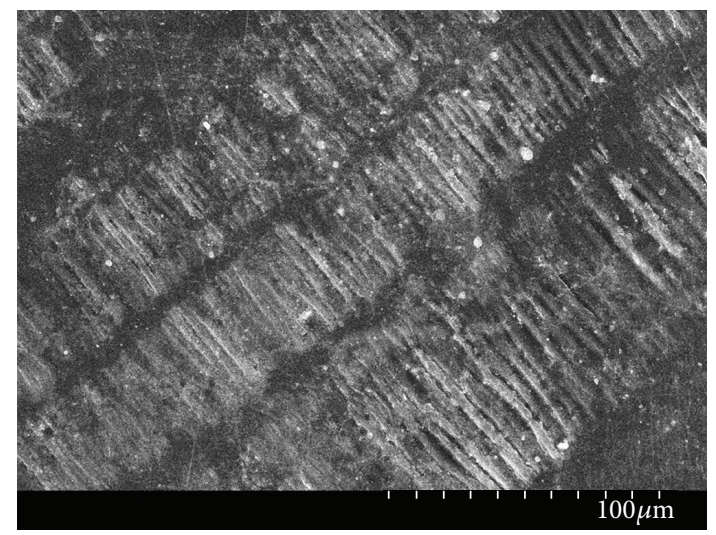

(d)

FIGURE 4: SEM image of the white pattern region of the ABS specimen after thermal aging at $260^{\circ} \mathrm{C}$ for (a) 0 min, (b) $10 \mathrm{~min}$, (c) $20 \mathrm{~min}$, and (d) $60 \mathrm{~min}$.

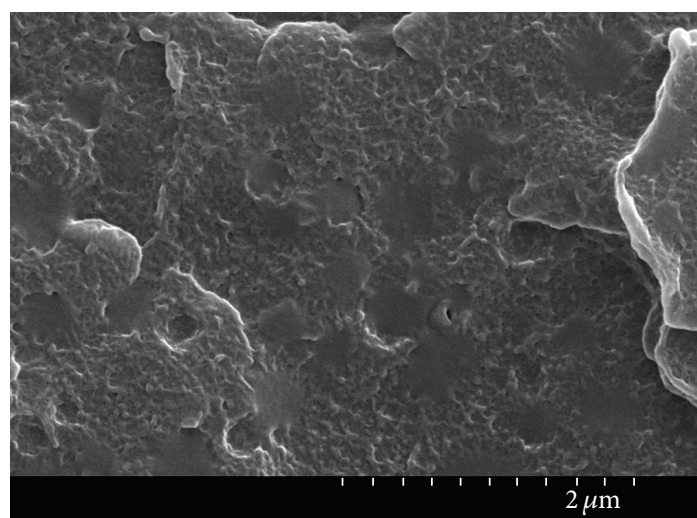

(a)

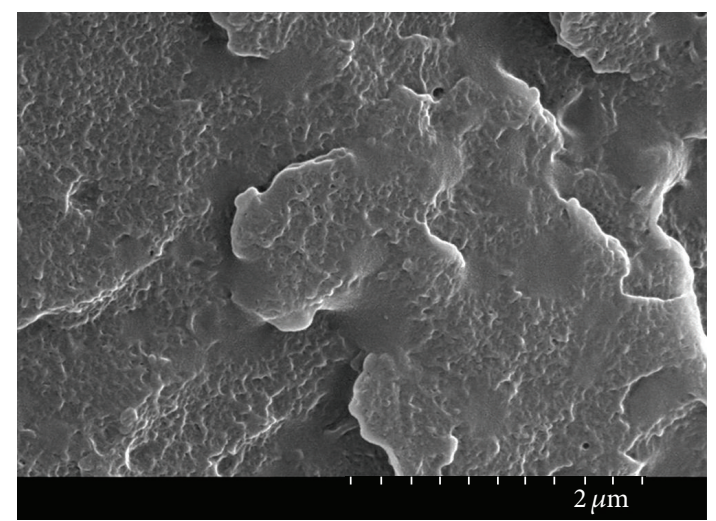

(b)

FIGURE 5: Fractured surface of the ABS specimen after hot pressing at (a) 220 and (b) $250^{\circ} \mathrm{C}$ for $20 \mathrm{~min}$.

$\left(200^{\circ} \mathrm{C}, 21.6 \mathrm{~kg}\right)$ and a heat distortion temperature of $85^{\circ} \mathrm{C}$ at $18.6 \mathrm{~kg} / \mathrm{cm}^{2}$ (Table 1). The values of Cross-WLF viscosity model parameters are $n=0.289, \tau^{*}=3.48 \times 10^{4} \mathrm{~Pa}, D 1=8.62$ $\times 10^{11} \mathrm{~Pa}-\mathrm{s}, T^{*}=373.15 \mathrm{~K}, A 1=24.96$, and $A 2=51.6 \mathrm{~K}$. The heat capacity, thermal conductivity, and thermal expansion were assumed to be constant at $1847 \mathrm{~J} / \mathrm{kg} .{ }^{\circ} \mathrm{C}, 0.192 \mathrm{~W} / \mathrm{m} \cdot{ }^{\circ} \mathrm{C}$, and $0.192 \times 10^{-5 \circ} \mathrm{C}^{-1}$, respectively.
The viscosity change of pristine ABS at different temperatures is presented in Figure 6. Viscosity data at temperature of $200^{\circ} \mathrm{C}$ has significantly lower values of viscosity in the whole range of shear rate compared to the flow behavior of ABS at $260^{\circ} \mathrm{C}$. It can be also found that shear viscosity of ABS decline with increasing shear rate for each temperature indicating a preservation of shear thinning behavior typical for a 


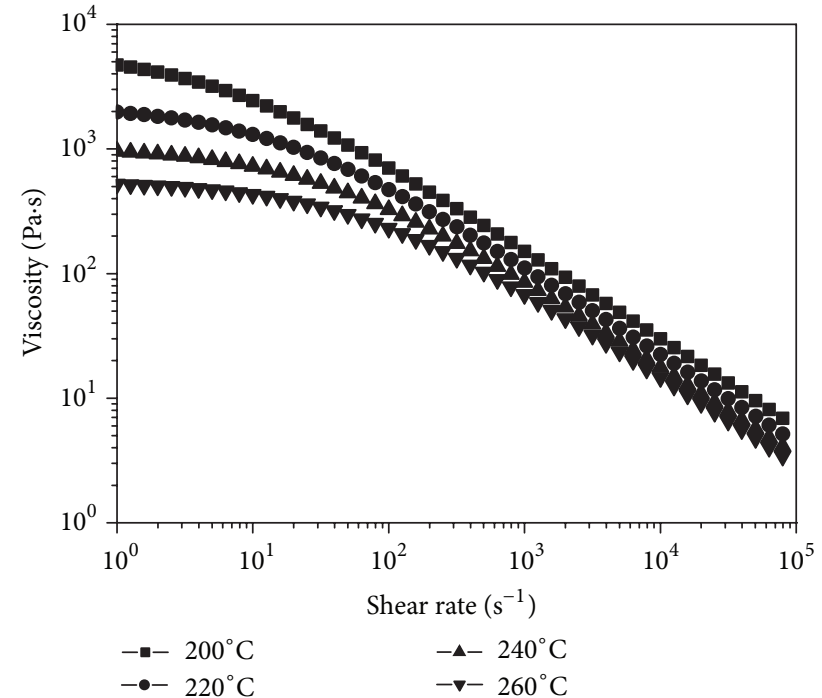

FIGURE 6: Viscosity change of pristine ABS at different temperatures.

common thermoplastic polymer. Thus, it can be concluded that the temperature increase in the tested ABS melt did not influence the shear thinning behavior while it effects only the magnitude of viscosity. Most polymers exhibit two regimes of flow behavior, the Newtonian and shear-thinning. The Newtonian flow occurs at low shear rates, but with increasing shear, the viscosity tends to fall away in what is termed shear-thinning behavior. The requirement viscosity model is very vital because it represents the observed behavior of polymer melts. These requirements that relate to viscositytemperature space are that the viscosity should decrease while the temperature is increasing. For the requirements that relate to viscosity-shear rate, the viscosity should decrease while the shear rate should increase. Therefore, these requirements must be applied to ABS on the filling phase and observe its behavior while melting.

3.4. PVT Behavior of ABS Resin. The PVT behavior of the ABS resin at 50,100,150, and $200 \mathrm{MPa}$ was demonstrated as in Figure 7. This PVT curve is used to obtain information about the compressibility and volumetric expansion of polymeric materials. The kink in the curve characterizes the glasstransition temperature $\left(T_{g}\right)$, which is a function of pressure. The $T_{g}$ is the temperature below which the physical properties of amorphous materials vary in a manner similar to those of a solid phase (glassy state) and above which amorphous materials behave like liquids (rubbery state), by compression.

The $T_{g}$ of former-liquid polymer is increased because of the compression. The slopes of the V-T curve denote the bulk thermal-expansion coefficients in the solid and liquid phases, respectively. This type of observations is very important in melt processing, especially where part consistency and minimum thermal shrinkage are important requirements; PVT relationships are used therefore to optimize the packing process cycle in injection molding. When the temperature of the material is increased, its specific volume (the inverse of

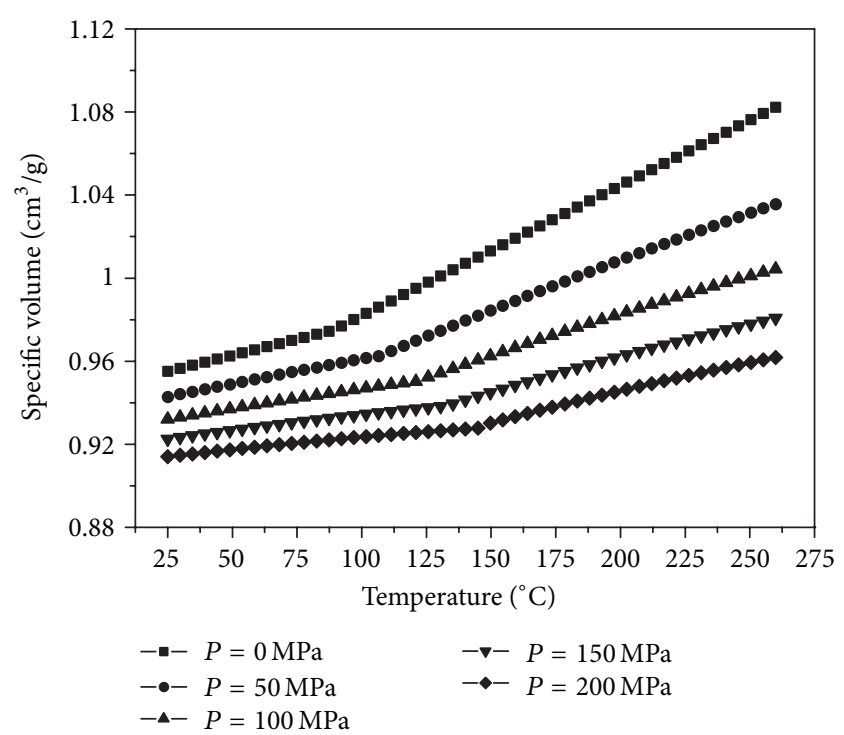

FIgURE 7: PVT behavior of the ABS resin.

density) also increases due to thermal expansion. The rate of increase becomes higher at the $T_{g}$, because the molecules have more freedom to move and they occupy more space. Above the $T_{g}$, the effect of chaining pressure has a significant effect on the specific volume-temperature relationships due to the compressible nature of high polymers; an increase in pressure decreased the specific volume at any given temperature.

3.5. Analysis of Injection-Molding Defects Using Moldflow Simulation. In order to analyse injection defects, Moldflow simulation was conducted using a single cavity mold with hot runner system. The processing parameters are shown in Table 2. The 3D-CAD drawing of cover mold was exported to the FE software using STEP file format and modeled using MF/FLOW module of Moldflow. The surfaces of part were converted to a midsurface mesh via Moldflow Preprocessing (Figure 8). The results of the converting were summarized in Table 4.

When designing plastic parts for the injection-molding process, the important element to understand is how the plastic is filling in the mold. In the mold injection filling stage, molten plastic is injected into the cavity until the cavity is just filled. As plastic flows into the cavity, the plastic in contact with the mold wall quickly freezes and this will create a frozen layer of plastic between the mold and the molten plastic.

Figure 9 shows the filling pattern of plastic flow for inferior cover part. The simulation was performed using measured thickness of inferior cover and design of runner gate for actual mold as shown in Figure 10(a).

Filling pattern results determine that all flow paths fill at the same time, balanced flow, hesitation, and over-packing. This result can be used to implement other parameter changes to improve filling such as, higher or lower injection rate, moving gate location and changes to the parts geometry. The color diagrams show the contour colors that represent 
TABLE 4: Converting results of the midsurface mesh via Moldflow preprocessing.

\begin{tabular}{lcc}
\hline Processing parameters & Unit & $\begin{array}{c}\text { Simulation } \\
\text { values }\end{array}$ \\
\hline Mesh Type & - & Midplane \\
Total number of nodes & No. & 23254 \\
Total number of injection location nodes & No. & 1 \\
The injection location node labels & - & 1 \\
Total number of elements & No. & 46428 \\
Number of part elements & No. & 46360 \\
Number of sprue/runner/gate elements & No. & 68 \\
Number of channel elements & No. & 0 \\
Number of connector elements & No. & 0 \\
Average aspect ratio of triangle elements & - & 2.0344 \\
Maximum aspect ratio of triangle elements & - & 16.7113 \\
Element number with maximum aspect ratio & - & 44141 \\
Minimum aspect ratio of triangle elements & - & 1.1553 \\
Element number with minimum aspect ratio & - & 6855 \\
Total volume & $\mathrm{cm}^{3}$ & 5447.5958 \\
Volume filled initially & $\mathrm{cm}^{3}$ & 245.5855 \\
Volume to be filled & $\mathrm{cm}^{3}$ & 5202.0103 \\
Part volume to be filled & $\mathrm{cm}^{3}$ & 5089.9284 \\
Sprue/runner/gate volume to be filled & $\mathrm{cm}^{3}$ & 112.0817 \\
Total projected area & $\mathrm{cm}^{3}$ & 6144.9301 \\
\hline
\end{tabular}

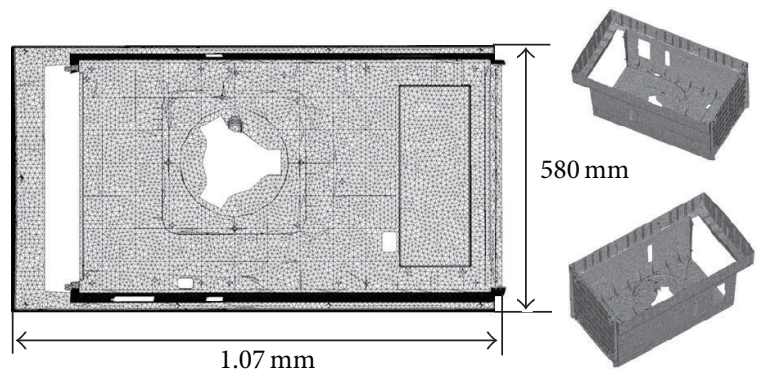

FIGURE 8: Result of the midsurface mesh conversion via Moldflow preprocessing.

the flow of plastic into the part. The result is dark blue at the start of the injection, and the last areas to fill are red. As the percentages of yellow and red increase, the difficulty in molding the part will increase and the part quality will decrease. If the part is a short shot, the section that did not fill has no color. As expected, the cover part filled very unequally and showed the formation of unfilled sections and burrs. The incomplete filling is one type of the defect that normally will appear either a lack of fluidity in the molten polymer or cross-section of mold that is too narrow. Hesitation can lead to asymmetrical and unpredictable flow patterns. It can reduce part quality due to variation in surface appearance, poor packing, high stresses, and nonuniform orientation of the polymer molecules. Alternatively, when the hesitation allows the flow front to freeze completely, part of the cavity may remain unfilled.
3.6. Two Different Case Studies. To avoid the actual mold defects, an other possible 5-gate system was simulated. The hot runners allowed the location of gates in the most favorable positions of cavities. In the procedure of injection molding, it is very important to determine the injection location inside a mold. It is because location at proper gate positions can minimize the flow hesitation at thin areas and this will be useful to avoid the occurrence of defects [21]. By designing the correct gating system, it is useful to have a thin section as the last point to fill, and so all the injection pressure is applied at this point. The configuration of each runner gate and thickness of components were shown in Figures 10(b) and 11(a), respectively.

Figure 12 shows the plastic flow filling pattern of modified 5 -gate system. This system had some drawbacks too. The left corner of cover part was very slowly filling and totally shows poor flow behavior. Unfilled section appeared because the component thickness was reduced from 3.2 to $3.0 \mathrm{~mm}$ in bottom directions. In fact, thin sections offer more resistance to flow than thick sections [3]. This can result in molten polymer in the thin section stopping or slowing significantly. Once the polymer starts to slow down, it will cool more rapidly, and so the viscosity will increase. This higher viscosity will inhibit flow further causing even faster cooling, and so the problem is self-propagating. Hesitation can occur in thin section of parts that have significant changes in wall thickness.

Based on the previous results, the use of 5-gate configuration was considered inadequate for product fabrication. The simulation study was extended to 6-gate system to eliminate unfilled sections. New identification numbers of each runner gate and thickness of component were shown in Figure 10(c) and Figure 11(b), respectively. The wall thickness of the injection-molded parts has an intense influence in its quality; the flow resistance is big when the wall thickness is too small, which result in unfilled defects in the cavity for large and complicated parts. This is because restricted areas in the mold, such as thin sections, small runners, and long flow lengths, require a larger pressure gradient and therefore a higher pressure to fill. ABS is a two-phase system; the rheology depends on the morphology and mutual interaction of the phases during the flow. Two different morphological models have been proposed to explain the rheological behavior of ABS flow behavior [22]. At lower shear rates, interfacial control is more pronounced where the deforming rubber particles embedded in SAN continuous phase. The rubber particles deform easily, so, various phenomena were observed. At higher shear rates, laminar deformational behavior dominates where both phases deform simultaneously and viscosity decreases rapidly, comparatively. SAN component behaved more Newtonian by increasing the temperature, which is usual for homogenous single phase thermoplastics [23]. Moreover, multiple gates are normally used for the fabrication of complex-shaped parts that all the cavities are filled simultaneously.

The achievement of balanced fill is to make the distance of the material travels from the sprue to each cavity the same. This approach will work as long as the material flows directly from the sprue to the gate of the part. An effective way of 


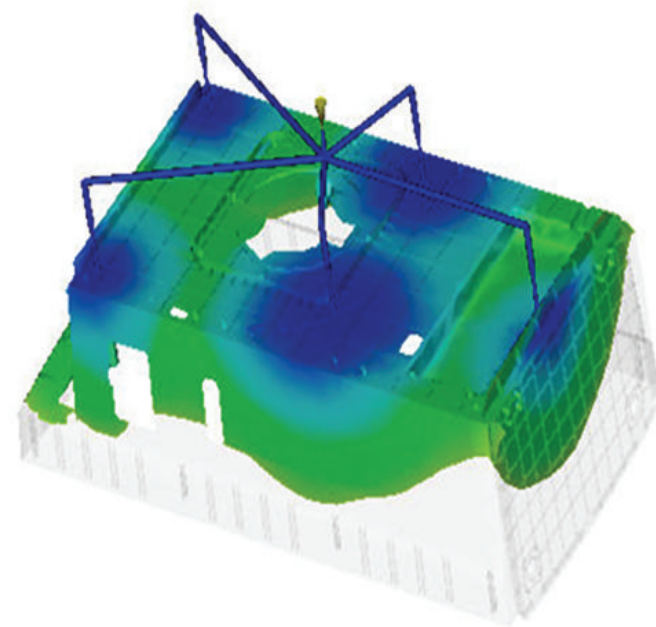

(a)

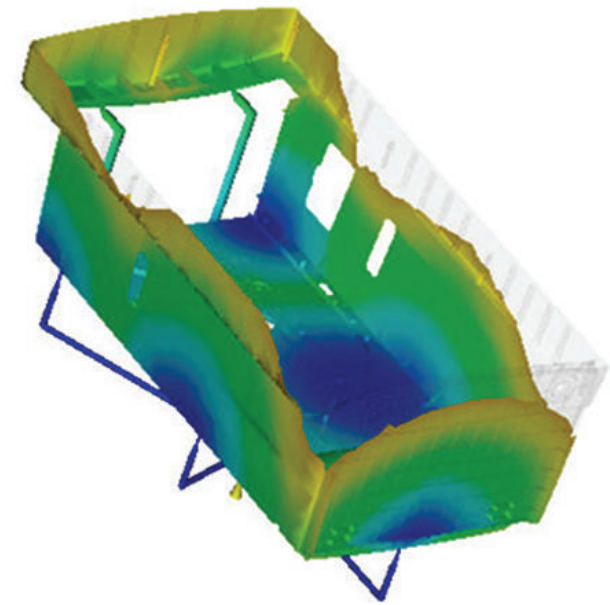

(b)

FIGURE 9: Filling pattern of the molten plastic flow for inferior cover part at (a) $70 \%$ filling and (b) $86 \%$ filling stage.

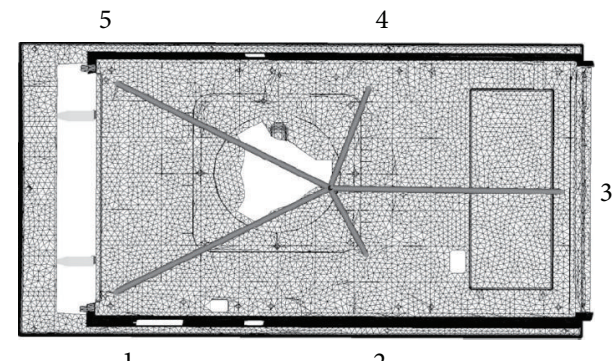

1

Gate1: $(-210,-400)$

Gate2: $(130,-66)$

Gate3: $(430,0)$

Gate4: $(200,70)$

Gate5: $(-210,-400)$

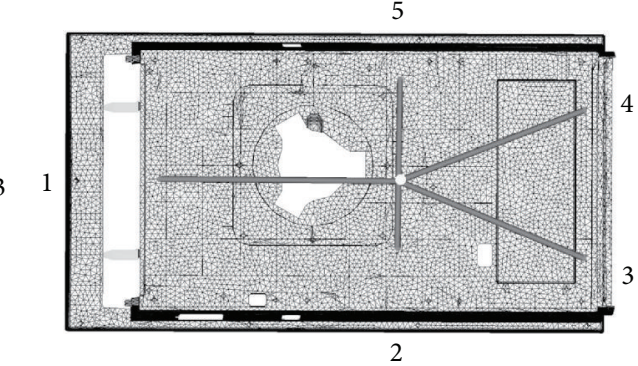

Gate1: $(-470,0)$

Gate2: $(130,0)$

Gate3: $(365,-150)$

Gate4: $(365,140)$

Gate5: $(0,200)$

(a)

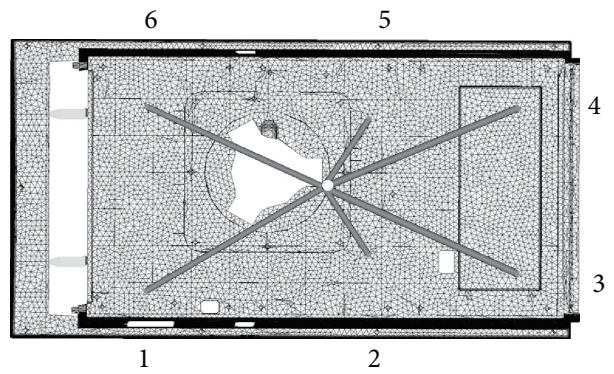

Gate1: $(-340,200)$

Gate2: $(80,-130)$

Gate3: $(365,-165)$

Gate4: $(365,155)$

Gate5: $(80,135)$

Gate6: $(-340,155)$

FIGURE 10: Three different hot runner gate systems ((a) actual gate, (b) 5-gate, and (c) 6-gate configurations). 

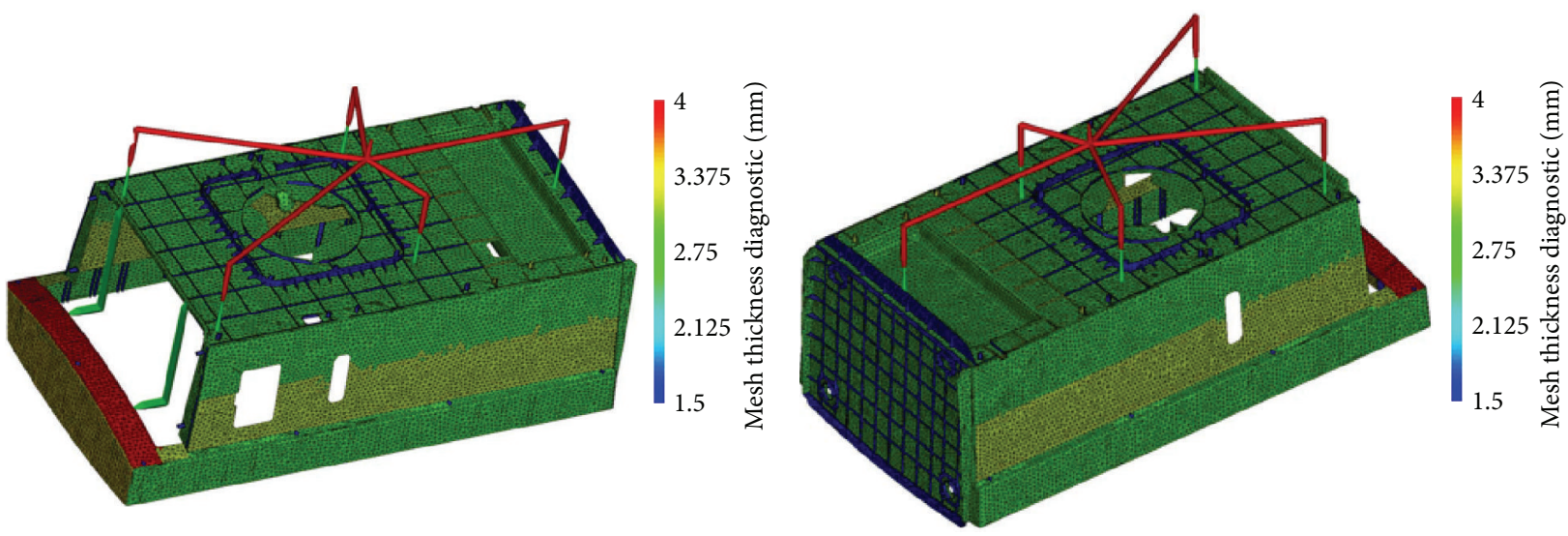

(a)
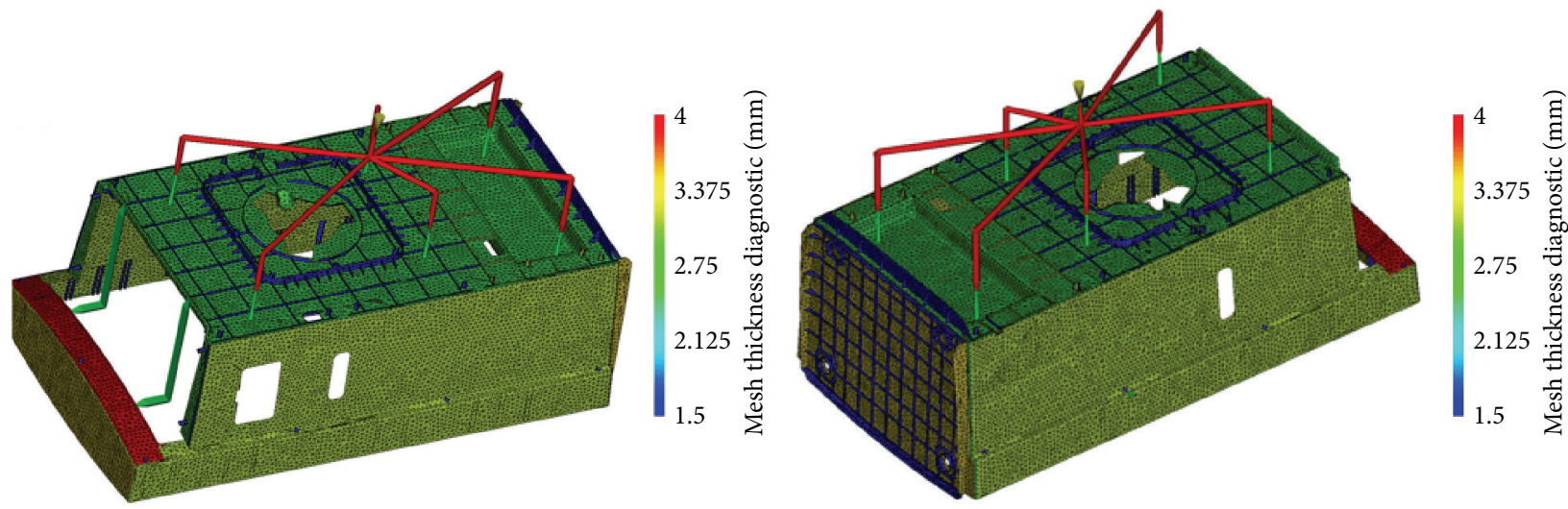

(b)

FIGURE 11: Thickness of the component for (a) 5-gate and (b) 6-gate system.

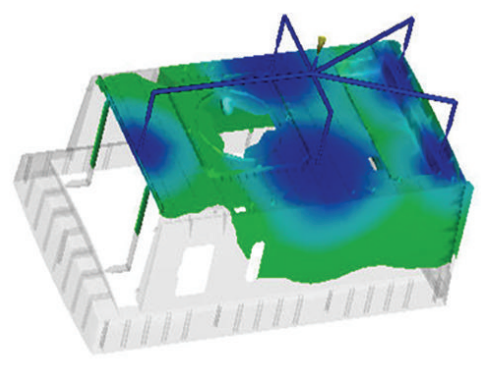

(a)

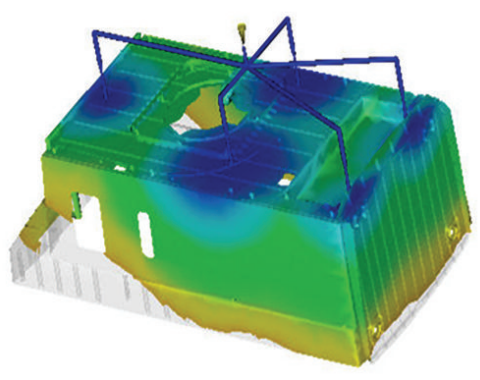

(b)

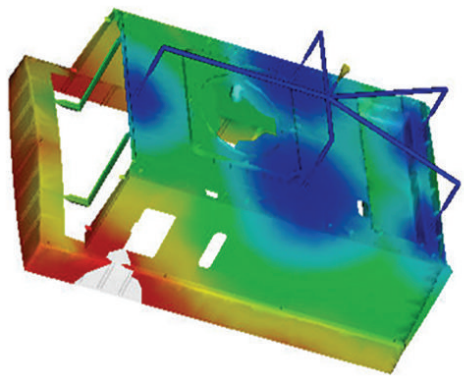

(c)

FIGURE 12: Plastic flow filling pattern of the 5-gate system at (a) 60\% filling, (b) $88 \%$ filling stage, and (c) $98 \%$ filling stage.

balancing the fill is to have one main runner that extends from the last cavity of one end of the mold to the last cavity on the opposite end, with subrunners feeding the individual cavities. It can be found that the filling pattern for 6-gate system is fairly uniform without any hesitation effect and all regions with the same color are filled simultaneously (Figure 13).

The injection pressure result in Figure 14, which is produced by a fill analysis, shows the maximum injection pressure value obtained before the velocity/pressure switchover occurs during the filling stage. At the beginning of filling, the pressure is zero in the absolute pressure scale throughout the mold. The pressure at a specific location starts to increase only after the melt front reaches that location. The pressure continues to increase as the melt front moves past, due to the increasing flow length between this specific location and the melt front. The color at each place on the model represents 


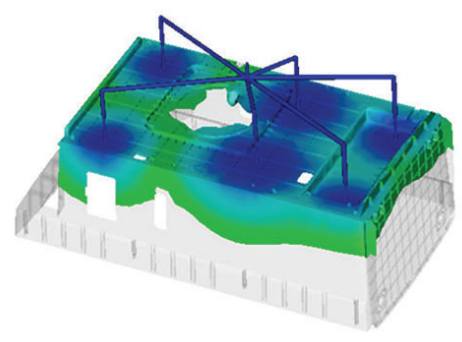

(a)

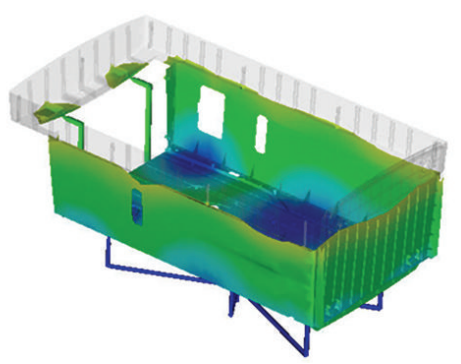

(b)

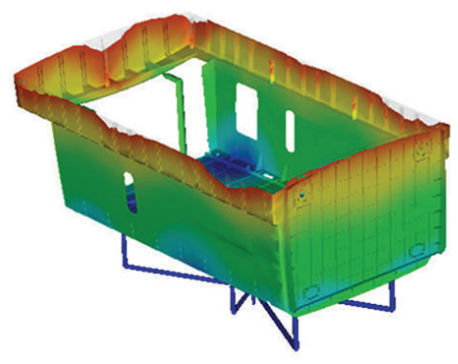

(c)

Figure 13: Plastic flow filling pattern of the 6-gate system at (a) 50\% filling, (b) 72\% filling stage and (c) $98 \%$ filling stage.

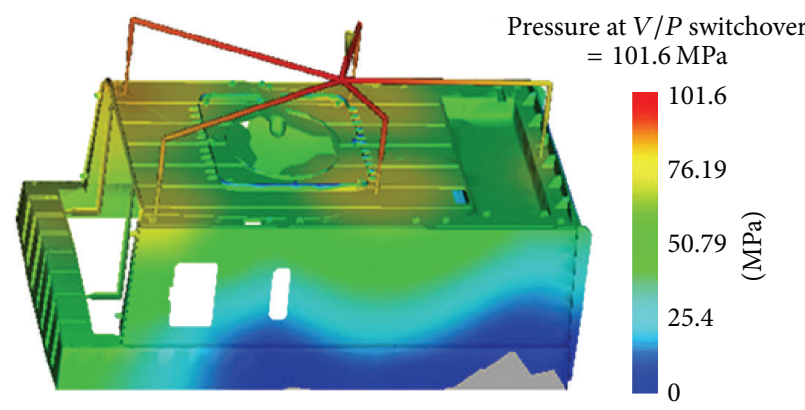

(a)

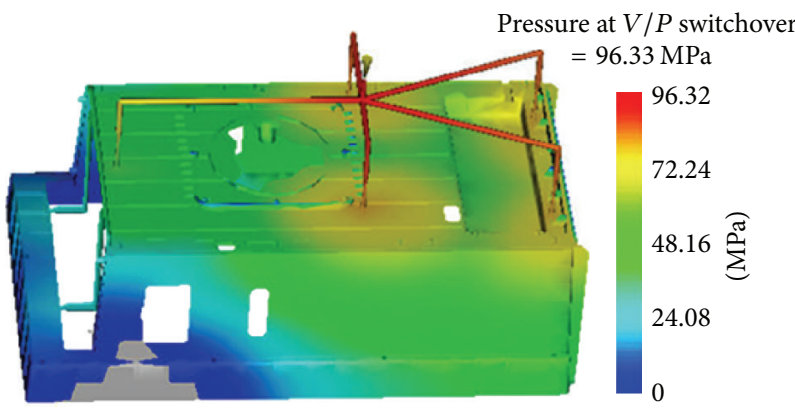

(b)

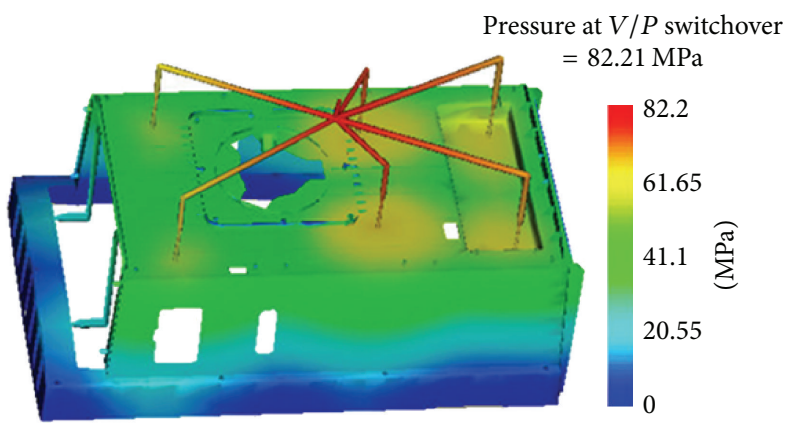

(c)

FIGURE 14: Results of the injection pressure analysis for three different gate systems ((a) actual gate, (b) 5-gate, and (c) 6-gate system).

the pressure at the place on the model. Two colors show the highest pressure (red) and lowest pressure (blue). The pressure difference from one location to another is the force that pushes the polymer melt to flow during filling. The pressure gradient is the pressure difference divided by the distance between two locations. The thermoplastic polymer always moves in the direction of the negative pressure gradient, from higher pressure to lower pressure; therefore, the maximum pressure occurs at the polymer injection locations and the minimum pressure occurs at the melt front during the filling stage. When applying modified 5-gate system, the maximum injection pressure reaches $96.3 \mathrm{MPa}$ at completely mold was filled. It appears that the injection pressure was decreased approximately $5.5 \%$ more than that of actual gate system (101.6 MPa). In case of 6-gate system, the maximum injection pressure reduced by $23 \%(82.3 \mathrm{MPa})$, and the injection pressure required to fill is well within the range of the molding equipment. The injection pressure can be used in conjunction with pressure drop result. Even if a section of a part has an acceptable pressure drop, the actual injection pressure in the same area may be too high. High injection can cause over-packing.

Figure 15 compares the clamping force changes of actual and simulated runner systems. The mold is closed within the platen arrangement and clamped using necessary force to hold the mold shut during the plastic injection cycle, thus preventing plastic leakage over the face of the mold. After the required cooling time, the mold is then opened by the clamping motor. In case of actual gate system, maximum clamping force is too high to properly operate on a 2,500-ton injection-molding machine. The maximum value will be over approximately $42 \%$ exceeding its capacity. In contrast, the maximum clamping force of 6-gate system (2,610 ton) was reduced about $37 \%$ and $19 \%$ than that of actual 


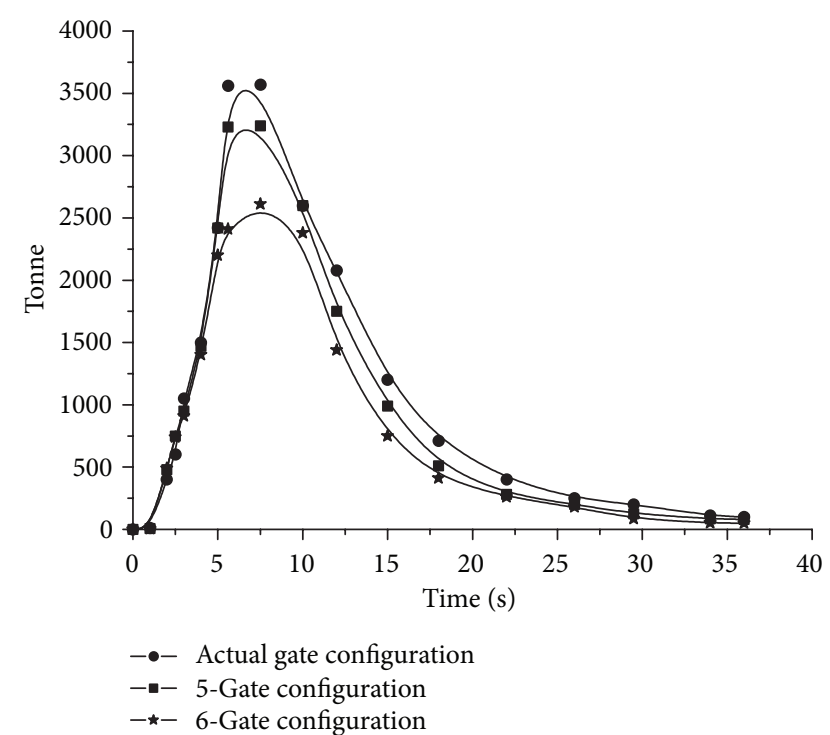

FIGURE 15: Clamping force changes of the actual gate, 5-gate, and 6-gate configurations.

gate (3,570 ton) and modified 5-gate system (3,240 ton), respectively. When the clamping force of the machine is exceeded, the molded parts stretch. This deformation causes wall thickness variation which results in both structural and cosmetic defects.

\section{Conclusion}

The large-sized cover part for air cleaner was injection molded with ABS resin using a 2,500-ton injection-molding machine. During manufacturing process, many undesired products with unfilled sections and burrs were formed repeatedly. Firstly, to determine the effect of processing temperature on these defects, the phase separation behavior, weight loss, and tensile properties of ABS resin were investigated. The tensile properties, weight loss, and phase separation of ABS resin were not significantly changed until $250^{\circ} \mathrm{C}$. These results indicated that the unfilled sections and burr defects were not originated in processing temperature. In order to analyse injection defects, Moldflow simulation was conducted using a single cavity mold with hot runner system. As revealed, inadequate mold design results to a poor plastic filling behavior and a decrease of the filling rate of polymer melts into the mold cavity. This increases injection pressure and leads to higher clamping force resulting in an unfilled sections and burrs. To improve these defects, possible two hot runner gate systems were designed and simulated using Moldflow program. The first configuration consisted of a single manifold with five runners, while the second configuration employed single manifold with six runners. The configuration with 5-gate system caused a higher injection pressure and clamping force than those of the configuration with 6-gate system. The second design avoided the problem of higher injection pressure and clamping force; hence, the defects of the actual mold as described have been solved.
This study provides a better understanding of the incomplete filling defects for large-size injection-molding parts and gives a better part quality and reduces rework and scrapping of materials.

\section{Conflict of Interests}

This work does not have a direct financial relation with the commercial identities mentioned in the paper that might lead to a conflict of interests for any of the authors.

\section{References}

[1] Z. B. Chen and L.-S. Turng, "A review of current developments in process and quality control for injection molding," Advances in Polymer Technology, vol. 24, no. 3, pp. 165-182, 2005.

[2] Y. K. Shen, P. H. Yeh, and J. S. Wu, "Numerical simulation for thin wall injection molding of fiber-reinforced thermoplastics," International Communications in Heat and Mass Transfer, vol. 28, no. 8, pp. 1034-1042, 2001.

[3] N. M. Neves, A. J. Pontes, and A. S. Pouzada, "Experimental validation of morphology simulation in glass fibre reinforced polycarbonate discs," Journal of Reinforced Plastics and Composites, vol. 20, no. 6, pp. 452-465, 2001.

[4] I. S. Dairanieh, A. Haufe, H. J. Wolf, and G. Mennig, "Computer simulation of weld lines in injection molded poly(methyl methacrylate)," Polymer Engineering and Science, vol. 36, no. 15, pp. 2050-2057, 1996.

[5] M.-C. Huang and C.-C. Tai, "Effective factors in the warpage problem of an injection-molded part with a thin shell feature," Journal of Materials Processing Technology, vol.110, no. 1, pp. 1-9, 2001.

[6] H. Shin and E.-S. Park, "Analysis of crack phenomenon for injection-molded screw using moldflow simulation," Journal of Applied Polymer Science, vol. 113, no. 4, pp. 2702-2708, 2009.

[7] F. Pisciotti, A. Boldizar, M. Rigdahl, and I. Ariño, "Effects of injection-molding conditions on the gloss and color of pigmented polypropylene," Polymer Engineering and Science, vol. 45, no. 12, pp. 1557-1567, 2005.

[8] S. Weir, "Predicting surface defects in injection molded PVC components," Journal of Vinyl and Additive Technology, vol. 16, no. 4, pp. 231-234, 1994.

[9] T. Boronat, V. J. Segui, M. A. Peydro, and M. J. Reig, "Influence of temperature and shear rate on the rheology and processability of reprocessed ABS in injection molding process," Journal of Materials Processing Technology, vol. 209, no. 5, pp. 2735-2745, 2009.

[10] X. Bai, D. H. Isaac, and K. Smith, "Reprocessing acrylonitrilebutadiene-styrene plastics: structure-property relationships," Polymer Engineering and Science, vol. 47, no. 2, pp. 120-130, 2007.

[11] B. H. Min, "A study on quality monitoring of injection-molded parts," Journal of Materials Processing Technology, vol. 136, no. 1, pp. 1-6, 2003.

[12] P. K. Kennedy, Practical and Scientific Aspects of Injection Molding Simulation, chapter 2, Technische Universiteit Eindhoven, Eindhoven, The Netherlands, 2008.

[13] J. Koszkul and J. Nabialek, "Viscosity models in simulation of the filling stage of the injection molding process," Journal of Materials Processing Technology, vol. 157-158, pp. 183-187, 2004.

[14] B. E. Tiganis, L. S. Burn, P. Davis, and A. J. Hill, "Thermal degradation of acrylonitrile-butadiene-styrene (ABS) blends," 
Polymer Degradation and Stability, vol. 76, no. 3, pp. 425-434, 2002.

[15] J. B. Adeniyi, "Clarification and discussion of chemical transformations involved in thermal and photo-oxidative degradation of ABS," European Polymer Journal, vol. 20, no. 3, pp. 291-299, 1984.

[16] M. G. Wyzgoski, "Effects of oven ageing on ABS," Polymer Engineering and Science, vol. 17, pp. 265-269, 1976.

[17] G.I. Öztürk, Selected methods of surface engineering applied to materials [M.D. thesis], Graduate School of Engineering and Natural Sciences, Sabanc University, 2004.

[18] J. K. Kim and C. K. Kang, "Basic studies on recycling of ABS resin," Polymer Plastics Technology and Engineering, vol. 34, no. 6, pp. 875-890, 1995.

[19] D. Salari and H. Ranjbar, "Study on the recycling of ABS resins: simulation of reprocessing and thermo-oxidation," Iranian Polymer Journal, vol. 17, no. 8, pp. 599-610, 2008.

[20] R. K. Jena, X. Chen, C. Y. Yue, and Y. C. Lam, "Viscosity of COC polymer (TOPAS) near the glass transition temperature: experimental and modeling," Polymer Testing, vol. 29, no. 8, pp. 933-938, 2010.

[21] H. S. Kim, J. S. Son, and Y. T. Im, "Gate location design in injection molding of an automobile junction box with integral hinges," Journal of Materials Processing Technology, vol. 140, no. 1-3, pp. 110-115, 2003.

[22] A. A. Qaiser, A. Qayyum, and R. Rafiq, "Rheological properties of ABS at low shear rates: effects of phase heterogeneity," Malaysian Polymer Journ, vol. 4, pp. 29-36, 2009.

[23] J.-Z. Liang, "Effects of extrusion conditions on rheological behavior of acrylonitrile-butadiene-styrene terpolymer melt," Journal of Applied Polymer Science, vol. 85, no. 3, pp. 606-611, 2002. 

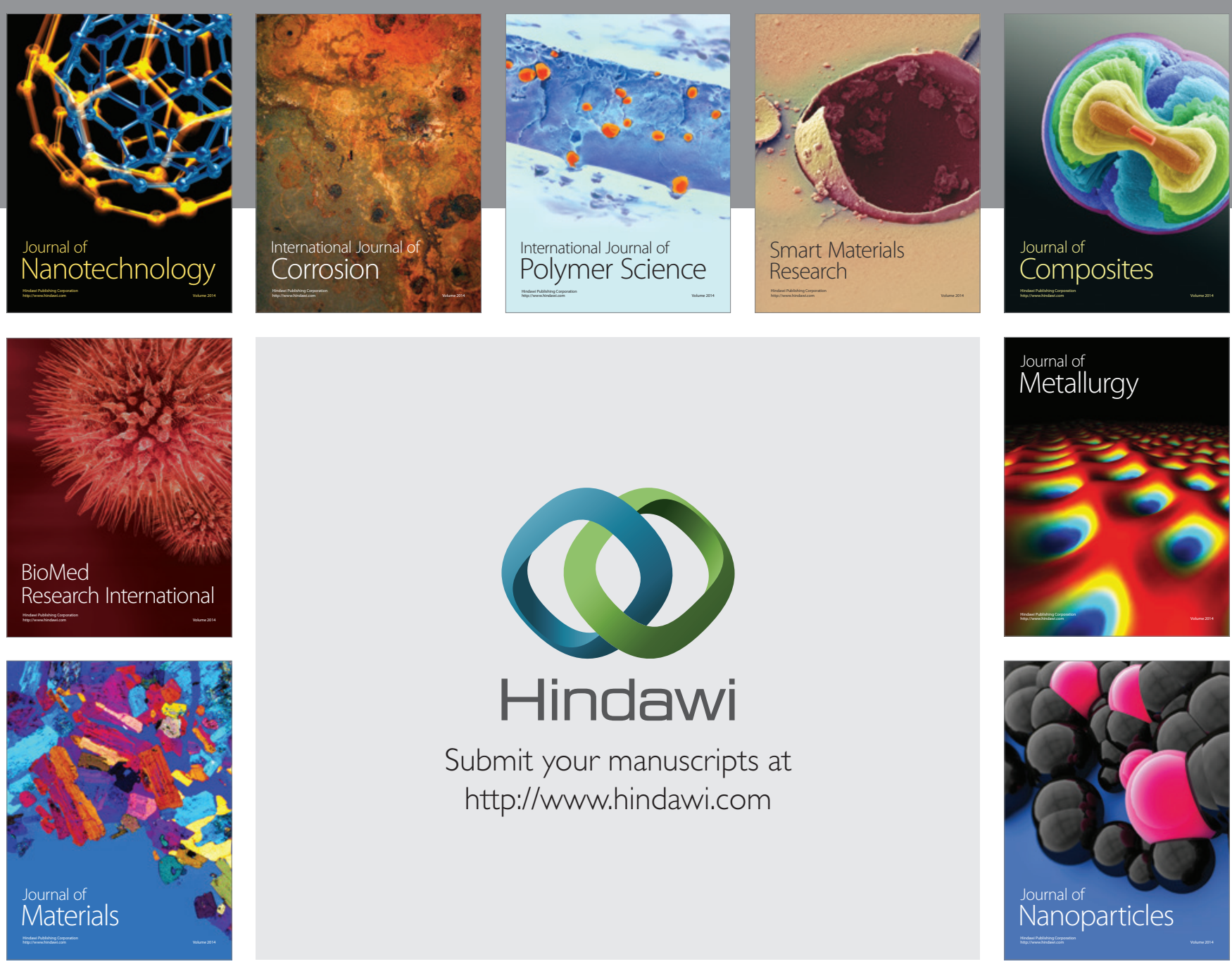

Submit your manuscripts at http://www.hindawi.com
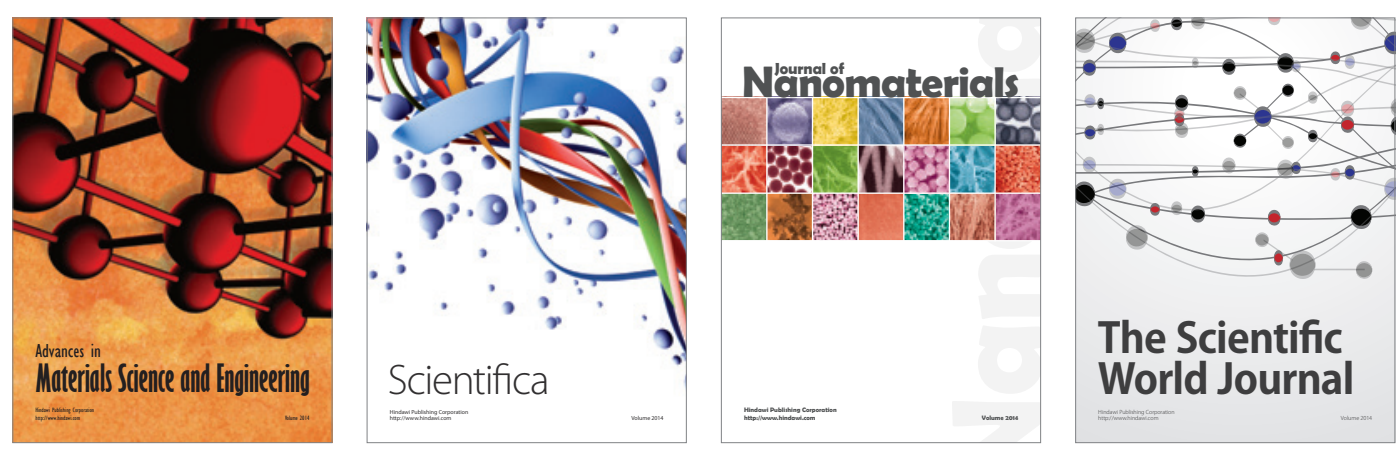

\section{The Scientific World Journal}
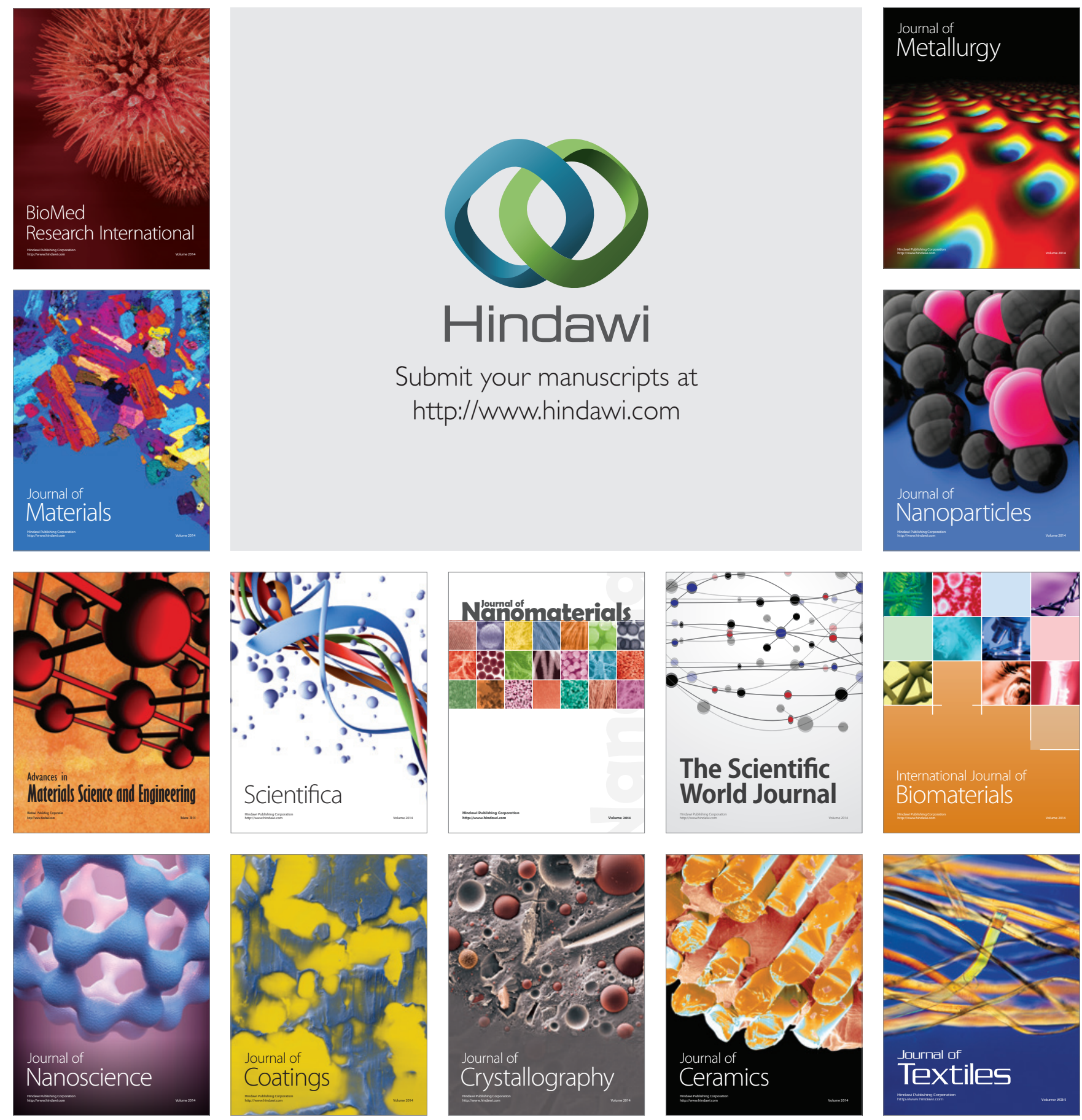